\title{
The Top 5 Papers of 2016-2017 for General Internists
}

By Kieran L. Quinn MD MSc FRCPC, Emily Hughes BScH, Amol A. Verma MD MPhil

DOI: $10.22374 /$ cjgim.v13i1.264

\begin{abstract}
About the Authors
Kieran L. Quinn and Amol A. Verma are with the Department of Medicine, University of Toronto, Toronto, ON, Canada. Emily Hughes and Amol A. Verma are with the Li Ka Shing Knowledge Institute, St. Michael's Hospital, Toronto, ON, Canada. Correspondence can be directed to: kieran.quinn@mail.utoronto.ca Submitted: January 1, 2018. Accepted January 2, 2018. Published March 5, 2018.
\end{abstract}

Each year, the Canadian Society for Internal Medicine (CSIM) Annual Meeting features a presentation of the "Top 5 Papers" in internal medicine (IM). We reviewed the publications in all major IM journals (New England Journal of Medicine, Journal of the American Medical Association, Lancet, Annals of Internal Medicine, British Medical Journal, and JAMA Internal Medicine) between November 2016 and October 2017 to identify 5 papers that we felt were particularly influential and important to practicing clinicians. These articles were all discussed on the free weekly podcast that we produce, The Rounds Table (http://healthydebate. ca/about-us/the-rounds-table). This article summarizes these "Top 5" papers.

\section{Source Citation}

Søndergaard L, Kasner SE, Rhodes JF, et al. Patent foramen ovale closure or antiplatelet therapy for cryptogenic stroke. N Engl J Med 2017;377:1033-42. doi:10.1056/NEJMoa1707404

\section{The Bottom Line}

The REDUCE trial was a multinational randomized controlled trial that enrolled younger participants with cryptogenic and non-lacunar stroke and demonstrated a reduction in recurrent clinical stroke with Patent Foramen Ovale (PFO) closure plus antiplatelet therapy compared to antiplatelet therapy alone. The number needed to treat was 28 to prevent one clinical stroke at 24 months.

\section{Methods}

Design: Open-label multinational randomized controlled trial. Setting: 63 sites in Canada, Denmark, Finland, Norway, Sweden, the United Kingdom, and the United States.
Participants: 664 participants aged 18-59 years (mean age 45 years) with recent cryptogenic and non-lacunar stroke (within 180 days). Participants were included if they had a PFO identified on trans-esophageal echocardiography with evidence of a right-toleft shunt. Participants receiving anticoagulation were excluded. Intervention: PFO closure via device insertion plus antiplatelet therapy compared to antiplatelet therapy alone. Antiplatelet therapy could be acetylsalicylic acid (ASA), ASA + dipyridamole, or clopidogrel.

Primary Outcome: Co-primary outcome of (1) Recurrent clinical ischemic stroke; (2) Composite of clinical stroke and radiological evidence of new brain infarction on magnetic resonance imaging at 24 months after enrolment. Participants were followed for 2 to 5 years (median 3.2 years).

Funding Source: Device manufacturer (W. L. Gore and Associates). 


\section{Main Results}

Table 1 - Rates of Recurrent Stroke

\begin{tabular}{|l|l|l|l|}
\hline \multicolumn{1}{|c|}{ Recurrent Clinical Ischemic Stroke* } & Hazard Ratio [95\% Cl] \\
\hline \multicolumn{1}{|c|}{$\begin{array}{c}\text { Antiplatelet therapy } \\
(n=441)\end{array}$} & \multicolumn{1}{|c|}{$\begin{array}{c}\text { PFO closure + antiplatelet therapy } \\
(n=223)\end{array}$} & \\
\hline No. of events (\%) & $6(4.1)$ & $12(5.4)$ & $0.23[0.09-0.62]^{* *}$ \\
\hline $\begin{array}{l}\text { Stroke rate (per } \\
100 \text { patient-years) }\end{array}$ & 0.39 & 1.71 & \\
\hline
\end{tabular}

* There was no difference in silent infarctions between groups (4.5\%)

${ }^{* *} p=0.002$

Table based on data from Søndergaard $L$ et al. ${ }^{1}$

\section{Limitations}

Premature discontinuation from the trial was noted in $14.8 \%$ of the antiplatelet-only group but only in $8.8 \%$ of the PFO closure group, resulting in differential follow-up between the groups. Prolonged cardiac monitoring was not a requirement for enrollment in the study, which could have resulted in undetected atrial fibrillation among participants. Because individuals requiring anticoagulation were excluded, this could modify the overall effect size. Participants and their treating physicians were not blinded.

\section{Commentary}

Approximately $25 \%$ of the population has a PFO. ${ }^{2}$ Previous RCTs found that PFO closure did not decrease the risk of recurrent stroke, ${ }^{3}$ whereas the REDUCE study and the CLOSE study (a similar RCT published in the same issue of $\mathrm{NEJM}^{4}$ ) found significant benefit from PFO closure as did long-term outcomes from the RESPECT trial. ${ }^{5}$ The main difference between the prior and most recent studies is in patient selection. These newer trials did not include individuals with lacunar strokes and the REDUCE trial did not include individuals using anticoagulation. In addition, most of the participants (82\%) in the REDUCE trial had a moderate-to-large right-to-left shunt. Thus, the most recent studies have been performed on a small subset of individuals with stroke who were deemed the most likely to benefit and their findings should not be considered generalizable outside of this population. These three recently published papers provide compelling evidence to suggest that in individuals who are younger than 60 with cryptogenic non-lacunar stroke and a $\mathrm{PFO}$ with moderate-to-large right-to-left shunt, $\mathrm{PFO}$ closure is effective in reducing recurrent clinical stroke.

\section{References}

1. Søndergaard L, Kasner SE, Rhodes JF, et al. Patent foramen ovale closure or antiplatelet therapy for cryptogenic stroke. N Engl J Med 2017;377:1033-42.
2. Meissner I, Whisnant JP, Khandheria BK, et al. Prevalence of potential risk factors for stroke assessed by transesophageal echocardiography and carotid ultrasonography: the SPARC study. stroke prevention: assessment of risk in a community. Mayo Clin Proc 1999;74(9):862.

3. Kent DM, Dahabreh IJ, Ruthazer R, et al. Device closure of patent foramen ovale after stroke: pooled analysis of completed randomized trials. J Am Coll Cardiol 2016;67:907-17.

4. Mas JL, Derumeaux G, Guillon B, et al. Patent foramen ovale closure or anticoagulation vs. antiplatelets after stroke. N Engl J Med 2017;377:1011-21.

5. Saver JL, Carroll JD, Thaler DE, et al. Long-term outcomes of patent foramen ovale closure or medical therapy after stroke. N Engl J Med 2017;377:1022-32.

\section{Source Citation}

Prandoni P, Lensing AWA, Prins MH, et al. Prevalence of pulmonary embolism among patients hospitalized for syncope. $\mathrm{N}$ Engl J Med 2016;375(16):1524-31. doi:10.1056/NEJMoa1602172.

\section{The Bottom Line}

The PESIT study was a multicenter cross-sectional study of individuals admitted to hospital for their first episode of syncope, which demonstrated that pulmonary embolism was present in $17 \%$ of these individuals.

\section{Methods}

Design: Prospective multicenter cross-sectional study.

Setting: 11 academic $(n=2)$ and community $(n=9)$ hospitals in Italy.

Participants: 560 participants aged $72-85$ years (mean age: 76 years) admitted to hospital with their first episode of syncope that was not due to seizure, stroke, or head trauma.

Exposure: First hospitalization for syncope.

Diagnostic Intervention: Participants were stratified into PE "likely" or "unlikely" (using both the Well's score and D-dimer 
assay within 48 hours of admission). CTPA was performed in those whom PE was "likely" according to Well's criteria (Well's $>4$ ) or with a positive D-dimer (D-dimer $>250-500 \mathrm{ug} / \mathrm{mL}$ ).
Primary Outcome: Proportion of individuals with confirmed PE. Funding Source: Institutional research funding, University of Padua.

\section{Main Results}

Table 1. Comparison of Diagnostic Yield by Investigation

\begin{tabular}{|l|c|c|}
\hline \multicolumn{1}{|c|}{ Investigation and Diagnosis of VTE } & $\begin{array}{c}\text { Individuals Receiving Test } \\
\text { No. (\%) [95\% Cl] }\end{array}$ & $\begin{array}{c}\text { Individuals With Positive Test Result } \\
\text { No. (\%) [95\% Cl] }\end{array}$ \\
\hline Any Investigation for VTE & $560(100)$ & $230(41.1)[37.1-45.2]$ \\
\hline D-dimer & $560(100)$ & $227(40.5)[36.6-44.7]$ \\
\hline CT Pulmonary Angiogram & $180(32.1)[28.4-36.1]$ & $72(40)[33.1-47.3]$ \\
\hline V/Q Scan & $49(8.8)[6.7-11.4]$ & $24(49.0)[35.6-62.5]$ \\
\hline Diagnosis of PE & $97(17.3)[14.2-20.5]$ & \\
\hline
\end{tabular}

$\mathrm{CT}=$ computed tomography; $\mathrm{PE}=$ pulmonary embolism; $\mathrm{V} / \mathrm{Q} \mathrm{Scan}=$ ventilation-perfusion scan; VTE = venous thromboembolism.

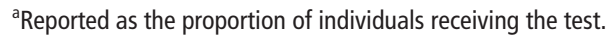

Table based on data from Prandoni P et al. ${ }^{1}$

\section{Limitations}

Several limitations of the PESIT study may have led to an overestimation of the prevalence of PE among individuals hospitalized with syncope, including: (1) the study did not include those who were discharged from the emergency; (2) individuals with prior syncope were excluded.

\section{Commentary}

The prevalence of PE in individuals who present with syncope is unknown, but the estimated prevalence of PE in hospitalized individuals is approximately $1.0 \% .^{2} \mathrm{~A}$ recent retrospective study using the same inclusion criteria as PESIT found the prevalence of venous thromboembolism among individuals hospitalized with syncope in Canada to be $1.4 \%{ }^{3}$ These differences raise questions about the generalizability of the PESIT findings, given the potential for selection bias, as different countries and healthcare systems may have differing thresholds for hospital admission as it relates to syncope.

The diagnostic methods for investigation of VTE are worthy of note. Forty percent of individuals with confirmed PE in this study had signs of a deep vein thrombosis. Therefore, a staged approach to investigation in clinical practice using compression ultrasonography may spare the potential harms associated with CTPA in some cases.

\section{References}

1. Prandoni P, Lensing AWA, Prins $\mathrm{MH}$, et al. Prevalence of pulmonary embolism among patients hospitalized for syncope. N Engl J Med 2016;375(16):1524-31.

2. Stein PD and Henry JW. Prevalence of acute pulmonary embolism among patients in a general hospital and at autopsy. Chest 1995;108(4):978-81.

3. Verma AA, Masoom H, Rawal S, et al. Pulmonary embolism and deep venous thrombosis in patients hospitalized with syncope: a multicenter cross-sectional study in Toronto, Ontario, Canada. JAMA Int Med 2017;177(7):1046-48.

\section{Source Citation}

Aaron SD, Vandemheen KL, FitzGerald JM, et al., Reevaluation of diagnosis in adults with physician-diagnosed asthma. JAMA 2017;317(3):269-79. doi:10.1001/jama.2016.19627

\section{The Bottom Line}

This multicenter prospective cohort study showed that 33\% of adults with physician-diagnosed asthma did not have the diagnosis on subsequent testing and almost all of them could be safely tapered off medications.

\section{Methods}

Design: Multicenter prospective cohort study.

Setting: 10 largest Canadian cities.

Participants: 613 adults over the age of 18 with physiciandiagnosed asthma in the last 5 years. Participants were recruited 
through random digit dialling. $87 \%$ of participants reported recently using asthma medications, $45 \%$ reported daily use of asthma medications. Participants were excluded if they were taking long-term oral steroids or had a greater than 10 pack year history of smoking.

Exposure: Physician-diagnosed asthma established within the past 5-years.

Diagnostic Intervention: Physician offices were contacted to determine how the initial diagnosis of asthma had been made in participants. Participants underwent a standardized diagnostic algorithm to determine the presence or absence of asthma, which involved spirometry (pre- and post-bronchodilator) over several visits and serial bronchial challenge tests. Participants requiring asthma medications had these gradually tapered off with serial testing. If spirometry was normal after stopping all asthma medications, participants were assessed by a respirologist. When asthma was ruled out, participants were followed for 12 months clinically and with bronchial challenge tests.

Primary Outcome: Proportion of participants in whom the diagnosis of asthma was ruled out. Participants were followed for 12 months.

Funding Source: The Canadian Institutes of Health Research. Methapharm Inc. supplied provocholine; Trudell Medical International Inc. supplied peak flow meters.

\section{Main Results}

Asthma was ruled out in 203 (33\%) participants. One hundred ninety-seven (97\%) of these individuals did not re-start asthma medications after 12 months. Asthma was ruled out in $26 \%$ of participants who reported daily asthma medication use.

Rates of asthma testing in the community were low. Only $51 \%$ of individuals with diagnosed asthma received formal testing. Participants with spirometry performed in the community on initial diagnosis were more likely to have asthma confirmed (Odds Ratio 1.79, 95\% CI 1.13-2.85). However, the results of spirometry at initial diagnosis were not always confirmed on repeat testing. Results for at least $16 \%$ of participants changed from negative to positive and at least $12 \%$ changed from positive to negative.

\section{Limitations}

Selection bias due to telephone recruitment and exclusion of individuals with corticosteroid use may have resulted in a population with less severe asthma.

\section{Commentary}

The results of this study may be explained by several mechanisms. Firstly, participants may have been initially misdiagnosed with asthma, particularly when formal spirometry testing was not performed. Secondly, the natural history of asthma may mean that the disease waxes and wanes over time. Thirdly, changes in environmental exposures over time could result in varying responses to spirometry and bronchial challenge testing.

This study highlights that spirometry should be part of asthma diagnosis, but it remains underutilized. Furthermore, even in individuals with physician-diagnosed asthma on regular medications, it may be worth revisiting the diagnosis. With careful monitoring, medications can be stopped safely for many individuals.

\section{Source Citation}

Felker GM, Anstrom KJ, Adams KF, et al. Effect of natriuretic peptide-guided therapy on hospitalization or cardiovascular mortality in high-risk patients with heart failure and reduced ejection fraction. JAMA 2017;318(8):713-718. doi:10.1001/ jama.2017.10565.

\section{The Bottom Line}

The GUIDE-IT trial was a multicenter randomized controlled trial of individuals in an ambulatory setting with heart failure and reduced ejection fraction (HFrEF). There was no difference in hospitalization for heart failure or cardiovascular mortality between participants who received an NT-proBNP-guided therapeutic strategy versus usual care.

\section{Methods}

Design: Unblinded multicenter randomized controlled clinical trial.

Setting: 45 clinical sites in the United States and Canada.

Participants: 894 individuals aged $51-72$ years (mean age: 63 years), with an LVEF $<40 \%$, elevated baseline NT-proBNP $>$ $2000 \mathrm{pg} / \mathrm{mL}$ or BNP $>400 \mathrm{pg} / \mathrm{mL}$, and a "HF event" within 1 year (defined as a prior ED visit or prior hospitalization for $\mathrm{HF}$, or outpatient use of IV diuretic therapy).

Intervention: Participants were randomized to either an NTproBNP-guided strategy to titrate pharmacologic therapy with the goal of achieving a target NT-proBNP of less than $1000 \mathrm{pg} /$ $\mathrm{mL}$ or usual care. Usual care was defined as guide-line directed therapy according to the most recent American Heart Association (AHA)/American College of Cardiology (ACC) practice guidelines for the management of heart failure. Physicians were encouraged to titrate neurohormonal therapies over diuretics unless participants were significantly volume overloaded or had evidence of pulmonary congestion. Participants were followed-up at 2 and 6 weeks, and then every 3 months thereafter, unless therapy doses were adjusted, in which case participants were seen 2 weeks following. 
Primary Outcome: Composite of time to first hospitalization or cardiovascular death.

Funding Source: National Institutes of Health; Roche Diagnostics provided support for NT-proBNP testing.

\section{Main Results}

The study was stopped early for futility when 894 of the planned 1100 patients had been enrolled after a median of 15 months of follow-up.

Table 1. Primary and Secondary Outcomes by Trial Arm

\begin{tabular}{|l|c|c|c|}
\hline & \multicolumn{2}{c|}{ Events at 1 Year No. (\%) } \\
\hline & NT-ProBNP-Guided Group $(n=446)$ & Usual Care Group $(n=448)$ & Hazard Ratio (95\%Cl) \\
\hline First Hospitalization or death & $151(33.8)$ & $161(36)$ & $0.98(0.79-1.23)$ \\
\hline Mortality & $66(15)$ & $77(17)$ & $0.86(0.62-1.20)$ \\
\hline CV Mortality & $53(12)$ & $57(13)$ & $0.94(0.65-1.37)$ \\
\hline First HF Hospitalization & $147(33)$ & $141(32)$ & $1.04(0.82-1.31)$ \\
\hline Total HF Hospitalizations & 350 & 277 & $1.29(0.97-1.72)$ \\
\hline
\end{tabular}

$\mathrm{CV}=$ cardiovascular; $\mathrm{HF}=$ heart failure.

Table adapted from Felker $\mathrm{G}$ et al. ${ }^{1}$

\section{Limitations}

Only $45 \%$ of participants achieved the target NT-proBNP levels of $<1000 \mathrm{pg} / \mathrm{mL}$. There were no between group differences in NT-proBNP levels throughout the trial, and both groups' levels decreased by $\sim 50 \%$ from baseline, thereby indicating effective, albeit similar care between groups.

\section{Commentary}

There are several potential reasons why differences were not found between the two trial arms. Participants in the usual care arm received excellent guideline-directed care as evidenced by the high use of proven therapies, and care was often provided in specialized heart failure centres, which may not reflect 'realworld' clinical practice.

Prior studies have found mixed evidence for the use of BNP to guide the management of $\mathrm{HF}^{2-4}$ These mixed results can largely be attributed to underlying study design issues, including small sample size and heterogeneity in participant selection. The GUIDE-IT trial is an appropriately powered, high quality study focusing on community-based older individuals with more advanced heart failure compared to previous trials, and supports the evidence that strictly using BNP to guide therapy doesn't achieve benefit over guideline-based care. Caution should be exercised in trying to apply the results of this study to the inpatient setting, where the evidence for use of BNP to guide treatment is less established.

\section{References}

1. Felker GM, Anstrom KJ, Adams KF, et al. Effect of natriuretic peptide-guided therapy on hospitalization or cardiovascular mortality in high-risk patients with heart failure and reduced ejection fraction. JAMA 2017;318(8):713-18. doi:10.1001/jama.2017.10565.

2. Eurlings LWM, van Pol PEJ, Kok WE, et al. Management of chronic heart failure guided by individual n-terminal pro-b-type natriuretic peptide targets: results of the PRIMA (Can PRo-Brain-natriuretic peptide guided therapy of chronic heart failure IMprove heart fAilure morbidity and mortality?) study. J Am Coll Cardiol 2010;56(25):2090-2100.

3. Troughton RW, Frampton CM, Yandle TG, et al. Treatment of heart failure guided by plasma amino terminal brain natriuretic peptide (N-BNP) concentrations. Lancet 2000;355(9210):1126-30.

4. Berger R, Moertl D,Peter S, et al. N-terminal pro-B-type natriuretic peptideguided, intensive patient management in addition to multidisciplinary care in chronic heart failure a 3-arm, prospective, randomized pilot study. J Am CollCardiol 2010;55(7):645-53.

\section{Source Citation}

Barnett ML, Olenski AR, and Jena AB. Opioid-prescribing patterns of emergency physicians and risk of long-term use. N Engl J Med 2017;376:663-73. DOI: 10.1056/NEJMsa1610524

\section{The Bottom Line}

This large observational study found that long-term opioid use was more common among individuals presenting to the Emergency Department (ED) who received a prescription from high-intensity prescribers than low-intensity prescribers. One new long-term opioid user occurred for every 48 new opioid prescriptions. 


\section{Methods}

Design: Retrospective observational study using administrative Medicare data.

Setting: All US emergency departments.

Participants: A 20\% random sample of US Medicare beneficiaries from 2008-2011 ( $n=377,629)$. Individuals were included if they visited the ED, were seen by an ED physician, and were not admitted to hospital. Individuals were excluded if they had filled an opioid prescription in 6 months prior to the ED visit. New opioid prescriptions were attributed to the ED visit if they were filled within 7 days after the ED visit.

Exposure: ED physicians were classified into quartiles from "low intensity" to "high-intensity" opioid prescribers compared to other
ED physicians within their own centre based on the proportion of ED visits for which an opioid prescription was filled.

Funding Source: National Institutes of Health.

Primary Outcome: Long-term opioid use by the patient, defined as 180 days or more of opioids dispensed in the 12 months after the ED visit (excluding the first 30 days after the ED visit).

\section{Main Results}

Individuals prescribed an opioid by high-intensity prescribers were more likely to have long-term opioid use (Table 1). Although causality cannot be established in this observational study, the authors estimated that, "for every 48 patients prescribed a new opioid in the ED who might not otherwise use opioids, 1 will become a long-term user."

Table 1. Rates of Opioid Prescribing and ED Encounters by Prescriber Intensity

\begin{tabular}{|c|c|c|c|}
\hline & $\begin{array}{l}\text { Low-Intensity } \\
\text { Prescriber } \\
(n=215,678)\end{array}$ & $\begin{array}{l}\text { High-Intensity } \\
\text { Prescriber } \\
(n=161,951)\end{array}$ & Adjusted OR [95\% Cl] \\
\hline Rates of opioid prescribing by physician* & $7.3 \%$ & $24.1 \%$ & $1.30[1.23-1.37]$ \\
\hline $\begin{array}{l}\text { Rates of any hospital encounter } 12 \text { months after index } \\
\text { ED visit* }\end{array}$ & $60.5 \%$ & $60.3 \%$ & $0.99[0.97-1.00]$ \\
\hline $\begin{array}{l}\text { Rates of opioid-related hospital encounters } 12 \text { months } \\
\text { after index ED visit* }\end{array}$ & $9.73 \%$ & $9.96 \%$ & $1.03[1.00-1.05]$ \\
\hline $\begin{array}{l}\text { Proportion of return ED visits where primary diagnosis } \\
\text { matched index ED visit }\end{array}$ & $5.61 \%$ & $5.68 \%$ & -- \\
\hline
\end{tabular}

* Rates were calculated as the proportion of ED visits where the event occurred divided by all ED visits for an individual prescriber

Table adapted from Barnett $\mathrm{M}$ et al. ${ }^{1}$

\section{Limitations}

It was not possible for the authors to determine why opioids were prescribed or if they were prescribed appropriately based on retrospective administrative data. This study only captured those patients insured under Medicare, all of whom are greater than 65 years of age, so the applicability to younger individuals is uncertain. Additionally, it is important to note that ED encounters account for only $5 \%$ of all opioids prescribed in the US.

\section{Commentary}

Opioid prescribing patterns varied widely (3-fold) among ED physicians within an individual centre. This translated to meaningful, but modest, individual differences in long-term opioid use $(0.35 \%$ absolute risk difference between high and low intensity prescribers). However, this aggregates to large population-level effects for such commonly used drugs. This study highlights that there are important long-term consequences of opioid prescribing in episodic care.

\section{Reference}

1. Barnett ML, Olenski AR, and Jena AB. Opioid-prescribing patterns of emergency physicians and risk of long-term use. N Engl J Med 2017;376:66373. DOI: $10.1056 /$ NEJMsa1610524 